\title{
Dysglycemia and Dyslipidemia Models in Nonhuman Primates: Part II. Model of Naturally Occurring or Experimental Obesity
}

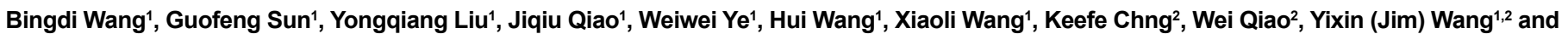 \\ Yong-Fu Xiao ${ }^{1 *}$
}

${ }^{1}$ Cardiovascular and Metabolic Disease Research, Crown Bioscience, Inc, 6 Beijing West Road, Taicang Economic Development Area, Taicang, Jiangsu Province, China ${ }^{2}$ CBNC, 150 N Research Campus Drive, Kannapolis, NC, USA

\begin{abstract}
Obesity is viewed as one of the most serious public health issues of this century, which is likely due to economic growth, urbanization, modernization, life-style change and decreased physical activity. In 2013, the American Medical Association classified obesity as a disease. Sustained excessive accumulation of body fat to the extent, such as overweight or obesity, can reduce life expectancy and increase health risks, particularly heart disease and type 2 diabetes. Cardiovascular, diabetes and other health problems of obesity have been studied for many decades. Inflammation, mitochondrial dysfunction, hyperinsulinemia, lipotoxicity, medical and others, such as genetic background and aging, may result in obesity. However, the underlying precise mechanisms have yet to be elucidated further. Various obesity animal models have been used and induced for obesity research and therapy. To better understand the pathophysiology of human obesity, corpulent nonhuman primates (NHPs) are useful models due to resembling humans, not only physiologically but in eating habits (bored-eat). NHP obesity models have been developed and used for delineating molecular and cellular mechanisms and for testing new novel therapies, which provides critical pre-clinic information for drug discovery. We recently published the data obtained from naturally occurring diabetes NHPs. This article summarizes the data collected from a large scale of naturally occurring and high calorie (fat) diet (HCD)-induced obesity monkeys housed in our facility. Manuscript for another NHP model, streptozocin-induced diabetes, developed in our facility will follow lately.
\end{abstract}

Keywords: Nonhuman primate; Cynomolgus monkey; Obesity model; High calorie diet; Diabetes

\begin{abstract}
Abbreviations: AAALAC: Association for Assessment and Accreditation of Laboratory Animal Care; BMI: Body Mass Index; DM: Diabetes Mellitus; DXA: Dual-Energy X-Ray Absorptiometry; FFA: Free Fatty Acid; FGF21: Fibroblast Growth Factor 21; FI: Food Intake; Hbalc: Hemoglobin A1c; HDL-C: High Density LipoproteinCholesterol; HCD: High Calorie (Fat) Diet; IACUC: Institutional Animal Care And Use Committee; LDL-C: Low Density LipoproteinCholesterol; NCD: Normal Calorie Diet; NHP: Non-Human Primate; TC: Total Cholesterol; TG: Triglycerides; WAT: White Adipose Tissue; Whtr: Waist-to-Height Ratio
\end{abstract}

\section{Introduction}

Obesity was classified as a disease by the American Medical Association in 2013 [1]. Generally speaking, obesity is defined when a person's body mass index (BMI, body weight (m) divided by the square of body height (h)) exceeds $30 \mathrm{~kg} / \mathrm{m}^{2}$. Obesity becomes a serious epidemic issue in many developed and developing countries. Economic growth, urbanization, societal changes and modernization have driven the obesity epidemic over recent decades. Globally, obesity has more than doubled since 1980. In 2014, more than 1.9 billion adults, 18 years and older, were overweight (BMI at $\left.25-30 \mathrm{~kg} / \mathrm{m}^{2}\right)$. Of these over 600 million were obese. $39 \%$ of adults aged 18 years and over were overweight in 2014, and 13\% were obese [2,3]. Excessive food intake plus lack/less physical activity are the main causes in most cases of obesity at single person level [4]. Some limited cases are primarily due to genetics, medical reasons or psychiatric illness [5]. Obesity can lead to several health problems, such as diabetes, cardiovascular diseases, obstructive sleep apnea, certain types of cancer, and osteoarthritis [6-8] and is one of the leading causes of morbidity and mortality. Obesity can result in insulin resistance and eventually develop to Type 2 diabetes mellitus. Several hypotheses for insulin resistance have been postulated and documented. Among those are inflammation, mitochondrial dysfunction, hyperinsulinemia and lipotoxicity $[9,10]$. Others, such as genetic background, aging, fatty liver, endoplasmic reticulum stress, hypoxia and lipodystrophy are the areas under active research for understanding mechanisms of insulin resistance [9]. The evidence connected to obesity-associated health comorbidities has mainly been obtained from epidemiological analyses of human subjects. Evidence shows that dyslipidaemia accelerates the atherosclerotic process and its morbid consequences. Nutraceuticals and functional food ingredients may potentially be beneficial to vascular health and reduce the overall cardiovascular risk from dyslipidemia [11]. However, the precise molecular and cellular mechanisms of obesity-associated health problems, including diabetes, have not yet been fully elicited. Various animal models have been used in research for understanding obesity/ diabetes mechanisms and discovery of novel therapies for the diseases [12-15]. Animal models can also be extended to determine if weight loss results from fat loss and/or from beneficial changes in key blood parameters (e.g. insulin). Typically, animal models have excellent predictive validity whereby drug-induced weight loss subsequently

${ }^{*}$ Corresponding author: Yong-Fu Xiao MD, PhD, Cardiovascular and Metabolic Diseases Division, Crown Bioscience, Inc. Science \& Technology Park, 6 Beijing Road, Taicang, Jiangsu Province, The People's Republic of China 215400 and Adjunct Professor, Department of Pharmacology and Physiology, Rutgers University-New Jersey Medical School, Newark, NJ, USA, Tel: +86-512-53879826; Fax: +86-512-5387-9801; E-mail: xiaoyongfu@crownbio.com

Received January 07, 2016; Accepted January 27, 2016; Published January 30 2016

Citation: Wang B, Sun G, Liu Y, Qiao J, Ye W, et al. (2016) Dysglycemia and Dyslipidemia Models in Nonhuman Primates: Part II. Model of Naturally Occurring or Experimental Obesity. J Diabetes Metab 7: 641. doi:10.4172/2155-6156.1000641

Copyright: $\odot 2016$ Wang B, et al. This is an open-access article distributed under the terms of the Creative Commons Attribution License, which permits unrestricted use, distribution, and reproduction in any medium, provided the original author and source are credited. 
translates to weight loss in man [16,17]. Currently, more studies related to obesity/diabetes research are moving toward using non-human primates (NHPs) which have better pathophysiological metabolism similarities to humans $[18,19]$. NHP models also play an important role in the screening of novel compounds for regulation of food intake, blood glucose and/or body weight, which can result in discovery and validation of new mechanism and therapeutic strategy or target [1921]. Compared with humans, NHPs are more accessible and feasible for biopsy and histopathology if there are scientific needs with IACUC approval.

This article introduces the characteristics of obesity models in our housed cynomolgus monkeys (Macaca fascicularis), naturally occurring and experimental induction, which have been highly valuable for research and treatment of dyslipidemia and dysglycemia [18-21]. Other NHP models, such as naturally occurring diabetes (see our recently published paper) and streptozocin-induced diabetes developed in our facility, are introduced elsewhere [22,23]. While no single model is necessarily applicable to every dysmetabolic research project and drug discovery, it has been suggested that certain models have great validity. Therefore, if possible, selective using of those preferable models to achieve the best potential outcome is recommended in order to address the very challenging disease, obesity. Normal and obese/diabetic monkeys housed in our animal center are collected periodically from the monkey farms in China. The monkeys are then raised in our own facility with a normal calorie diet (NCD) of monkey chow for naturally occurring obesity model (Beijing Keao Xieli Feed Co., LTD, Beijing, China) or a high calorie (fat) diet (HCD) for experimental model with enrichment of seasonal fruits and vegetables in accordance with the Association for Assessment and Accreditation of Laboratory Animal Care (AAALAC) regulations and guidelines [22,24-26]. All the procedures for sampling or data collection used in this study were approved by the Institutional Animal Care and Use Committee (IACUC) (Crown Bioscience, Inc., Taicang, Jiangsu province, The People's Republic of China) [22,24-26].

\section{Naturally occurring obesity model in NHPs}

General characteristics: As humans and other great apes (Hominoidea) diverged from Old World monkeys (Cercopithecoidea) $[27,28]$, obesity and diabetes occur in Old World monkeys, which would thus provide a good research model to study human obesity and diabetes [29]. Monkeys raised in indoor cages increase the chance to become obesity and facilitate their development to obesity-associated diseases in an age-dependent manner when given food ad libitum [28,30-32]. Like humans, these monkeys develop to Type 2 diabetes mellitus and other complications, such as nephropathy, orthmopathy, neuropathy and cardiovascular complications $[18,28,33]$.

In order to clarify the various groups of obesity level, the criteria were set up for grouping our in-housed monkeys $(n=103)$ from lean to morbidly obese mainly based on their total fat levels (Table 1). Four groups of the cynomolgus macaques: lean, chubby, obese and morbidly obese are listed in Table 1. Evidence shows that lean monkeys housed in our facility had total fat $\leq 10 \%$ measured with dual-energy $\mathrm{x}$-ray absorptiometry (DXA) scan (GE Model: Lunar DPX-NT, Milwaukee, WI, USA) [34]. Other monkeys could be grouped to various obese groups based on their total fat \% levels (Table 2). DXA is recognized as the best method to measure the body fat composition. Before DXA scan, animal was ensured without dosed radioactive nuclide and/or radiopaque drug within recent 5 days, and also all materials, such as neck ring and/or jacket which could reduce X-ray penetration, were removed from the animal. The DXA machine went through a QA test before scanning the first animal each day. Each animal with overnight fasting was anesthetized initially with ketamine $10 \mathrm{mg} / \mathrm{kg}$ i.m. and then $5 \mathrm{mg} / \mathrm{kg}$ i.m. as needed. Its height, body weight and waist circumference were measured for calculation of the body mass index (BMI). The animal was laid dawn on its back and a DXA scan was started. Two $\mathrm{X}$-ray beams with different energy levels were aimed to various organs and tissues. The scanned results were be collected and analyzed with the software loaded to the DXA machine by the manufacture (GE Model: Lunar DPX-NT, Milwaukee, WI, USA).

The general metabolic characteristics of the grouped monkeys being housed and being fed with the normal calorie diet (Beijing Keao Xieli Feed Co., LTD, Beijing, China) in CrownBio animal facility are shown in Table 2. Clearly, compared with the lean monkeys, the monkeys with various increases in body fat in the obese groups were relatively older and showed significantly increases in their body weights. In addition, the waist circumference, BMI, WHtR (waist-toheight ratio) and total fat (\%) were also significantly $(p<0.05)$ increased in the dysmetabolic groups (Table 2). In contrast, the leptin levels in the obese and morbidly obese groups were significantly lower $(p<0.05)$ compared with the lean group. However, most of other parameters were not significantly different among 4 groups (Table 2 ).

\section{Distribution and correlation in naturally obese monkeys}

To examine the distribution of obese levels of our in-housed monkeys, the animal numbers were plotted against various total body

\begin{tabular}{|c|c|}
\hline Grading & Total Fat (\%) \\
\hline Lean & $\leq 10 \%$ \\
\hline Chubby & $>10, \leq 20 \%$ \\
\hline Obese & $>20, \leq 35 \%$ \\
\hline Morbidly Obese & $>35$ \\
\hline
\end{tabular}

Table 1: Grading the monkeys into various groups based on their body fat composition levels.

\begin{tabular}{|c|c|c|c|c|}
\hline & Lean & Chubby & Obese & Morbidly Obese \\
\hline Parameter & $\begin{array}{c}\mathrm{n}=30(\mathrm{M} / \mathrm{F} \\
29 / 1)\end{array}$ & $\begin{array}{c}\mathrm{n}=26(\mathrm{M} / \mathrm{F}, \\
25 / 1)\end{array}$ & $\begin{array}{c}\mathrm{n}=42(\mathrm{M} / \mathrm{F}, \\
39 / 3)\end{array}$ & $\mathrm{n}=5(\mathrm{M} / \mathrm{F}, 5 / 0)$ \\
\hline Age (yr) & $11.5 \pm 0.85$ & $13.7 \pm 0.67^{*}$ & $14.1 \pm 0.35^{*}$ & $12.3 \pm 2.13$ \\
\hline Body weight (kg) & $7.2 \pm 0.39$ & $9.3 \pm 0.38^{*}$ & $10.6 \pm 0.28^{*}$ & $13.6 \pm 1.44^{*}$ \\
\hline Height $(\mathrm{cm})$ & $80.0 \pm 1.08$ & $82.6 \pm 1.06$ & $84.0 \pm 0.70^{*}$ & $82.1 \pm 1.19$ \\
\hline $\begin{array}{c}\text { Waist } \\
\text { circumference } \\
\text { (cm) }\end{array}$ & $35.6 \pm 1.36$ & $44.0 \pm 1.03^{*}$ & $50.3 \pm 0.74^{*}$ & $62.5 \pm 4.57^{*}$ \\
\hline BMI $\left(\mathrm{kg} / \mathrm{m}^{\wedge} 2\right)$ & $11.1 \pm 0.37$ & $13.6 \pm 0.37^{*}$ & $15.0 \pm 0.27^{*}$ & $20.0 \pm 1.65^{*}$ \\
\hline WHtR & $0.44 \pm 0.01$ & $0.53 \pm 0.01^{*}$ & $0.60 \pm 0.01^{*}$ & $0.76 \pm 0.05^{*}$ \\
\hline Total fat $(\%)$ & $5.2 \pm 0.31$ & $15.1 \pm 0.50^{*}$ & $26.6 \pm 0.69^{*}$ & $43.9 \pm 2.78^{*}$ \\
\hline Glucose (mg/dL) & $144 \pm 20.4$ & $122 \pm 18.5$ & $112 \pm 12.5$ & $77 \pm 8.4$ \\
\hline Insulin (mIU/mL) & $59 \pm 13.2$ & $254 \pm 121.7$ & $223 \pm 67.6$ & $107 \pm 47.0$ \\
\hline C-peptide (nmol/L) & $1.35 \pm 0.25$ & $1.80 \pm 0.21$ & $1.84 \pm 0.15$ & $1.98 \pm 0.93$ \\
\hline $\mathrm{TG}(\mathrm{mg} / \mathrm{dL})$ & $146 \pm 35.7$ & $168 \pm 46.1$ & $143 \pm 20.6$ & $78 \pm 11.1$ \\
\hline $\mathrm{TC}(\mathrm{mg} / \mathrm{dL})$ & $141 \pm 6.7$ & $127 \pm 7.5$ & $119 \pm 6.2^{*}$ & $136 \pm 5.1$ \\
\hline HDLc (mg/dL) & $50 \pm 4.2$ & $48 \pm 3.1$ & $50 \pm 2.4$ & $65 \pm 5.4$ \\
\hline LDLc (mg/dL) & $55 \pm 3.9$ & $42 \pm 3.4^{*}$ & $46 \pm 3.7$ & $49 \pm 6.0$ \\
\hline Leptin (ng/mL) & $11.7 \pm 0.22$ & $9.9 \pm 0.86$ & $9.8 \pm 0.41^{*}$ & $10.6 \pm 0.24^{*}$ \\
\hline $\mathrm{CRP}(\mathrm{mg} / \mathrm{L})$ & $2.7 \pm 0.28$ & $3.0 \pm 0.79$ & $1.7 \pm 0.45$ & $2.2 \pm 0.40$ \\
\hline WBC (109/L) & $11.5 \pm 1.21$ & $11.3 \pm 1.16$ & $12.1 \pm 0.69$ & $13.2 \pm 1.54$ \\
\hline
\end{tabular}

Note: M/F, male/female; BMI, body mass index=body weight/height ${ }^{\wedge} 2 ; \mathrm{WHtR}$ waist-to-height ratio=waist circumference/height; ${ }^{*}, p<0.05$, versus lean

Table 2: General characteristics of the grouped animals. 
fat \% levels (Figure 1A). According to the criteria listed in Table 1, 29\% of the monkeys were lean $(n=30)$ and $25 \%$ were chubby $(n=26)$. One big portion $(41 \%)$ of the monkeys were obese $(n=42)$ and only $5 \%$ of them were morbidly obese $(n=5$, Figure $1 B)$.

Most of these studied monkeys are currently alive and housed in our animal facility for diabetes and dyslipidemia research. To examine the correlations between obese levels and some specific parameters, several key results obtained from the monkeys were plotted against the total body fat $\%$ levels (Figure 2). In the experimental animals the total body fat $\%$ levels measured with DXA assay were not significantly correlated with the ages (Figure 2A), but were significantly correlated with their body weights (Figure 2B) and BMIs (Figure 2D). The total body fat $\%$ levels measured with DXA assay were also significantly correlated with the waist circumferences (Figure 2E) and WHtRs (Figure 2F), but not with the heights (Figure 2C).

\section{Experimental obesity model in NHPs}

Experimental obese animal models are often induced via feeding with high calorie (fat) diet (HCD). To investigate obesity and dysmetabolism in NHPs, the following groups were fed with the HCD (Table 3). The $1^{\text {st }}$ group ( $\mathrm{n}=134 \mathrm{HCD}+18 \mathrm{NCD}$ control) was lean with normoglycemia; the $2^{\text {nd }}$ group included the NHPs with normoglycemia $(\mathrm{n}=8)$, pre-DM $(\mathrm{n}=11)$ and DM $(\mathrm{n}=11)$; the $3^{\text {rd }}$ group included 6 normal lean NHPs; and the $4^{\text {th }}$ group $(n=10)$ was already obese with the

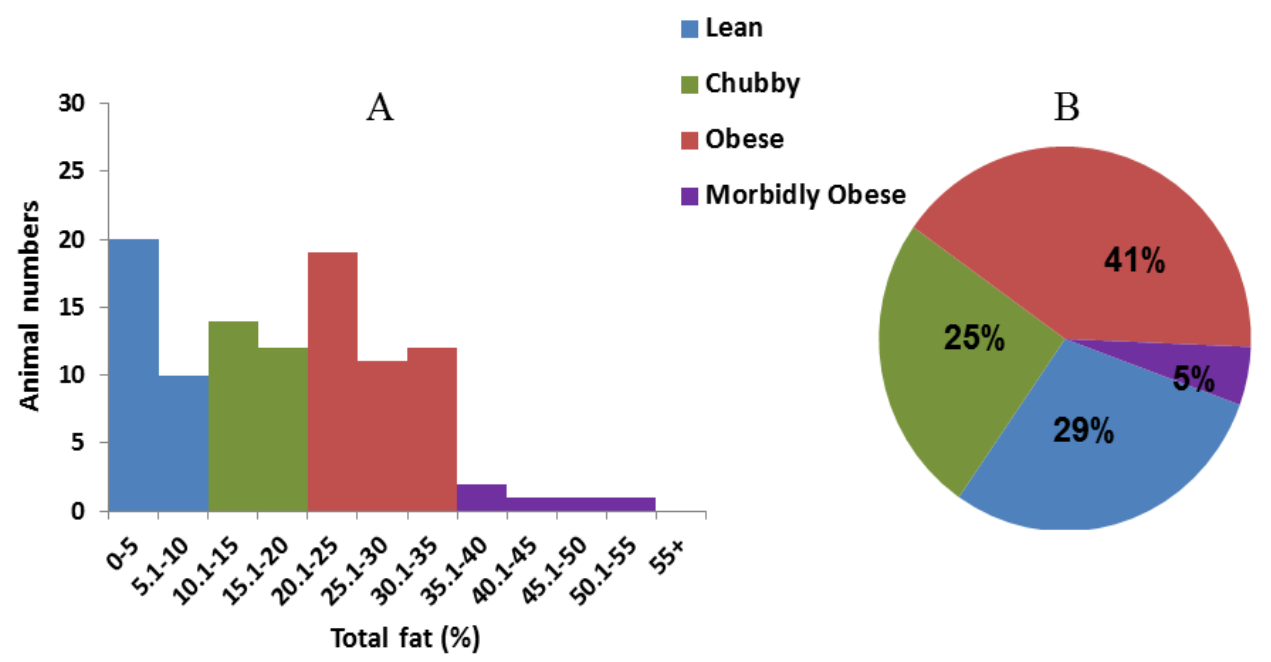

Figure 1: Distribution $(\boldsymbol{A})$ and percentage $(\boldsymbol{B})$ of the normal and obese monkeys in the studied natural obesity monkeys ( $\mathrm{n}=103$ ) based on their total fat percentages and criteria of obesity listed in Table 1.
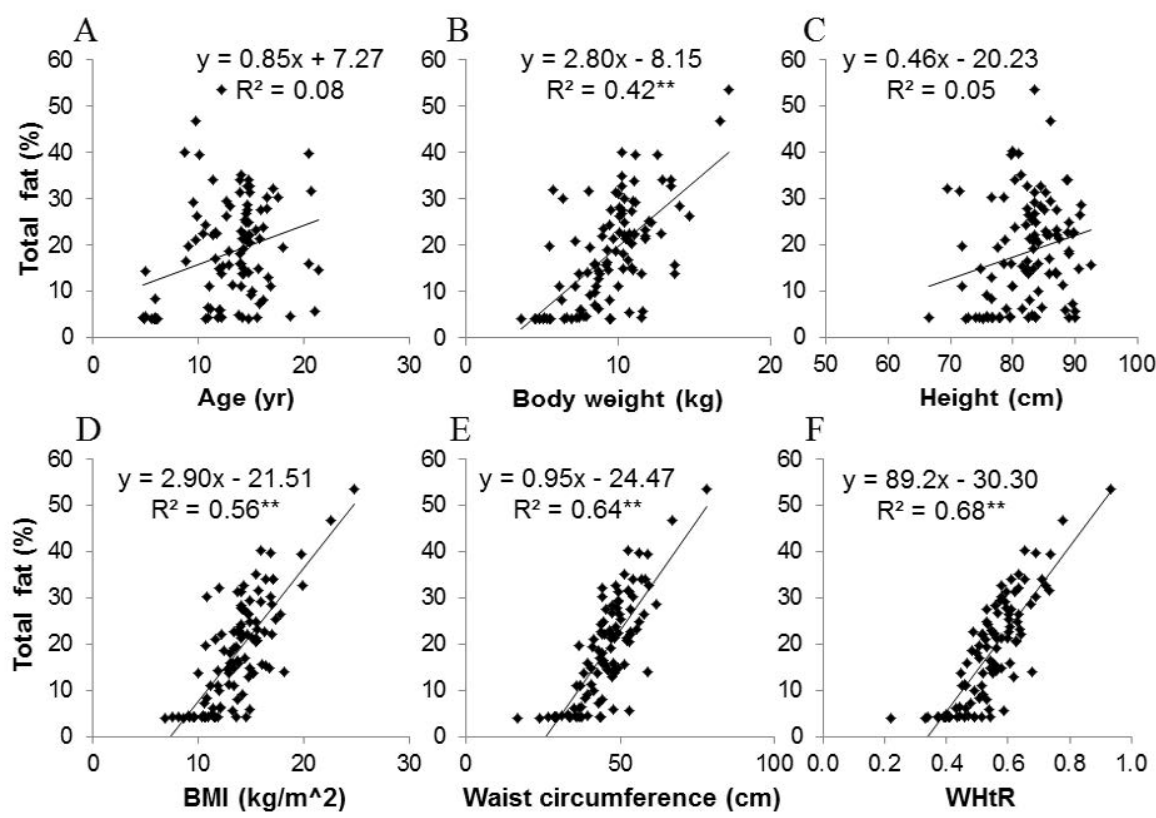

Figure 2: Correlations of multiple parameters in obese cynomolgus monkeys. $\boldsymbol{A}, \boldsymbol{B}$, and $\boldsymbol{C}$, The correlations between the total body fat \% and age, body weight, or height are shown, respectively. The total body fat percentages were significantly correlated to the body weights $(\boldsymbol{B})$, but not to ages $(\boldsymbol{A})$ or heights $(\boldsymbol{C}, p>0.05)$. The total body fat percentages were also significantly correlated to $(\boldsymbol{D})$ BMls, waist circumferences $(\boldsymbol{E})$, and $\mathrm{WHtR}(\boldsymbol{F}) .{ }^{* *}, p<0.01$. 
Citation: Wang B, Sun G, Liu Y, Qiao J, Ye W, et al. (2016) Dysglycemia and Dyslipidemia Models in Nonhuman Primates: Part II. Model of Naturally Occurring or Experimental Obesity. J Diabetes Metab 7: 641. doi:10.4172/2155-6156.1000641

Page 4 of 11

\begin{tabular}{|c|c|c|c|c|c|c|c|c|c|}
\hline \multicolumn{9}{|c|}{ Nutrient composition (\%) } & \multicolumn{2}{c|}{ Energy source (\%) } \\
\hline Protein & Fat & Fiber & Calcium & Phosphate & Cholesterol & Protein & Fat & Carbohydrate \\
\hline$\geq 16.3$ & $\geq 17.7$ & $\geq 1.9$ & 1.1 & 0.6 & $\geq 0.5$ & 16.2 & 39.5 & 44.3 \\
\hline
\end{tabular}

Table 3: Nutrient compositions and energy sources of the high calorie (fat) diet (HCD).

A

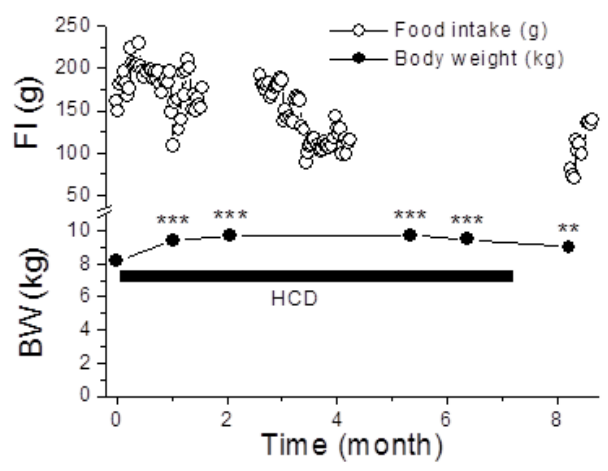

$\mathrm{C}$

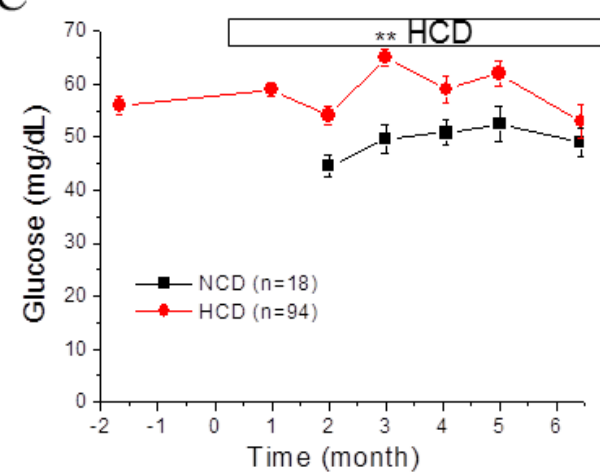

$\mathrm{B}$

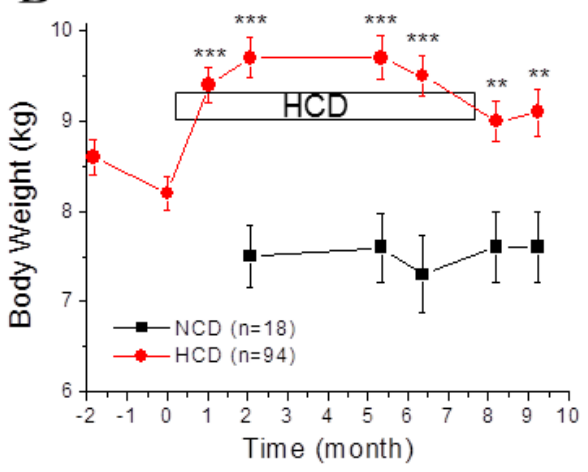

$\mathrm{D}$

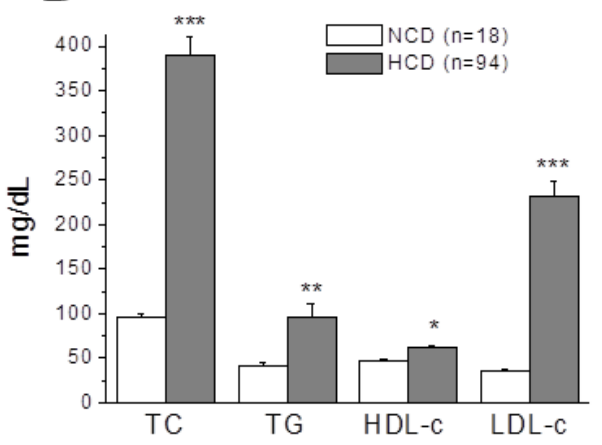

Figure 3: The effects of the HCD on food intake, body weight, serum glucose and lipids. $\boldsymbol{A}$, The changes of food intake (upper panel) and body weight (low panel) in normal cynomolgus monkeys $(n=94)$ fed with the HCD. B. The body weight changes in the normal NHPs fed with NCD only $(\boldsymbol{\square}, n=18)$ or with NCD and then HCD $(\bullet$, $\mathrm{n}=94)$. $\boldsymbol{C}$, The changes of serum glucose in the normal NHPs fed with NCD only $(\boldsymbol{\bullet}, \mathrm{n}=18)$ or with HCD $(\bullet, \mathrm{n}=94)$. $\boldsymbol{D}$, Comparisons of serum TC, TG, HDLc and LDLC in NCD-only NHPs (blank bars, $\mathrm{n}=18$ ) with those fed with HCD NHPs for over 7 months $(\bullet, \mathrm{n}=94)$. BW, body weight; $\mathrm{FI}$, food intake. *, $p<0.05 ;{ }^{* \star}, p<0.01 ;{ }^{* \star \star}, p<0.001$; vs. pre-HCD baseline or vs. control group in panel $\boldsymbol{D}$.

total fat $>23 \%$, but with normoglycemia. The observation and results are detailed and summarized below.

\section{HCD-induced obesity in lean and normoglycemia NHPs}

Lean normal monkeys with normoglycemia were enrolled in this study. A total of 94 male cynomolgus monkeys with the initial body weight of $8.2 \pm 0.18 \mathrm{~kg}$ (mean \pm SE, Figure $3 \mathrm{~A}$ ) and age of $12.3 \pm 0.1$ years (from 10.7 to 14.0 years old) were selected for feeding with the HCD (Table 3 ). Compared with its pre-HCD level, body weight was significantly increased during HCD period $(p<0.001)$ and even still significantly higher 2 months after termination of $\mathrm{HCD}(p<0.01$, Figure $3 \mathrm{~B})$. The daily food intake (FI) was gradually increased from around $150 \mathrm{~g} /$ day at the beginning to 200 $\mathrm{g} /$ day in the $1^{\text {st }} 2$ months and then decreased to $100 \mathrm{~g} / \mathrm{kg} 3$ months after feeding with the HCD (Figure $3 \mathrm{~A}$ upper panel). After termination of HCD the food intake was low with the amount of $80 \mathrm{~g} /$ day and then gradually recovered toward the pre-HCD level (Figure $3 \mathrm{~A}$ upper panel). Another group of similar lean normal monkeys with normoglycemia $(\mathrm{n}=18)$ was enrolled into the study as the control group at the time of 2 months after HCD started in the HCD group (Figure 3B). The data clearly show that the body weight of the NCD group was relatively stable and no significant increase during more than 4-month observation (Figure 3B,).
To examine the effects of the HCD on blood glucose and lipids, the animals were fasted overnight for around 16 hours and then their blood samples were collected for the assays. Serum glucose levels showed some variability during HCD in HCD-treated group (Figure $3 \mathrm{C}, \bullet$ ) and also in the NHPs of NCD group (Figure 3C, $\mathbf{\square}$ ). The variability in NCD group was not statistically significant. In contract, compared with NCD group, the lipid profiles of TC, TG, HDLc and LDLc were significantly increased in the animals fed with the HCD (Figure 3D). Among those changes, TC and LDLc were even more obvious. These results suggest that relatively longterm HCD can cause significant increase in body weight, TC, TG, HDLc and LDLc, but may affect blood glucose moderately as a whole group.

To test the biological effects of the HCD with a relatively short feeding duration, another group of lean NHPs $(n=40$, Figure 4) with normoglycemia was fed with the HCD for a period of 100 days which was about half of the time in the study described above (Figure 3). Compared to the pre-HCD levels, the body weight, serum glucose, HbAlc, TC and LDLc increased significantly after 100-day HCD (Figure 4). However, the changes of TG and HDLc were not so obvious. The data indicate that some parameters, such as BW, TC and LDLc, responded to a relatively short duration of HCD are more obvious and sensitive. 


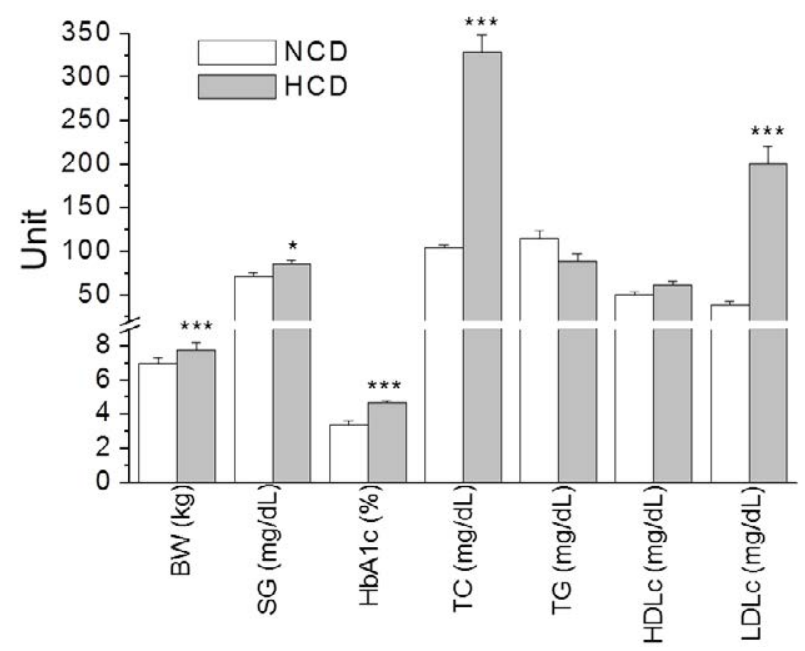

Figure 4: The effects of the HCD on body weight, serum glucose, $\mathrm{HbA} 1 \mathrm{c}$ and lipids in the normal NHPs fed with the HCD for over 4 months. Compared with the pre-HCD baseline levels (blank bars, NCD), the body weight, serum glucose, $\mathrm{HbA1c}, \mathrm{TC}$, and LDLc significantly increased 4 months after feeding with the HCD (grey bars, $n=40$ ). However, TG and HDL did not significantly change $(\mathrm{n}=40)$. ${ }^{*}, p<0.05 ;{ }^{* *}, p<0.01 ;{ }^{* * *}, p<0.001$; vs. pre-HCD baseline.

\begin{tabular}{|c|c|c|c|}
\hline \multirow{2}{*}{ Item } & Normal & Pre-DM & DM \\
\cline { 2 - 4 } & $8(\mathrm{M} / \mathrm{F}, 7 / 1)$ & $11(\mathrm{M} / \mathrm{F}, 9 / 2)$ & $11(\mathrm{M} / \mathrm{F}, 6 / 5)$ \\
\hline Age $(\mathrm{y})$ & $10 \pm 1.2$ & $10 \pm 1.1$ & $16 \pm 1.1^{\text {*\# }}$ \\
\hline Body weight $(\mathrm{kg})$ & $7.4 \pm 1.1$ & $8.5 \pm 1.3$ & $7.4 \pm 0.5$ \\
\hline Serum glucose $(\mathrm{mg} / \mathrm{dL})$ & $69 \pm 2.4$ & $93 \pm 2.2^{*}$ & $144 \pm 13.2^{\text {*\# }}$ \\
\hline Serum insulin $(\mu \mathrm{ll} / \mathrm{mL})$ & $38 \pm 16$ & $72 \pm 34$ & $216 \pm 140$ \\
\hline Blood HbA1c $(\%)$ & $4.6 \pm 0.1$ & $4.5 \pm 0.1$ & $6.3 \pm 0.6^{\text {*\# }}$ \\
\hline Serum C-peptide $(\mathrm{nmol} / \mathrm{L})$ & $3.5 \pm 2.6$ & $1.5 \pm 0.3$ & $2.3 \pm 0.6$ \\
\hline Serum TC $(\mathrm{mg} / \mathrm{dL})$ & $127 \pm 10$ & $128 \pm 12$ & $118 \pm 10$ \\
\hline Serum TG $(\mathrm{mg} / \mathrm{dL})$ & $71 \pm 17$ & $85 \pm 11$ & $122 \pm 17$ \\
\hline Serum HDLc $(\mathrm{mg} / \mathrm{dL})$ & $61 \pm 5$ & $58 \pm 4$ & $53 \pm 6$ \\
\hline Serum LDLc $(\mathrm{mg} / \mathrm{dL})$ & $43 \pm 6$ & $48 \pm 8$ & $37 \pm 4$ \\
\hline
\end{tabular}

*, $p<0.05$, versus normal; \#, $p<0.05$, versus Pre-diabetes.

Table 4: Baseline glycemic and lipidemic parameters before high calorie diet.

\section{HCD-induced obesity in diabetes NHPs}

To investigate the potential differences of HCD effects in animals with various blood glucose levels, the NHPs with normoglycemia $(n=8)$, pre-DM $(n=11)$ and DM $(n=11)$ were fed with the HCD. The defining criteria for normoglycemia, pre-DM and DM have been described elsewhere [35]. Table 4 shows the baseline data of some glycemic and lipidemic parameters in the grouped animals before feeding with the $\mathrm{HCD}$. Clearly, the age, serum glucose and HbAlc were significantly higher in the DM group compared with the normal and pre-DM animals. The serum glucose level was also significantly higher in preDM group than normal one (Table 4). However, the other parameters, such as serum insulin, C-peptide, TC, TG, HDLc and LDLc were not significantly different among the three groups (Table 4 ).

To examine HCD effects in all the animals (male, $n=30$ ) enrolled in this study, the whole group results were pulled together. Body weight, serum glucose, TC and LDLc were significantly increased 50 days after HCD (Figure 5, $p<0.001$ ). The changes of other parameters, such as HbA1c, insulin, C-peptic, TG, and HDLc, were not statistically significant (Figure 5, $p>0.05$ ).

To look at the effects of the HCD in individual groups, the body weights were compared. The baseline body weight of the pre-DM animals $(n=11)$ was higher than normal $(n=8)$ and $D M(n=11)$ groups, but the differences did not reach statistical difference (Figure 6A, NCD). Body weight increased in all three groups 50 days after HCD, but the increases did not reach statistical significance compared with NCD (Figure 6A, HCD). Compared with the normal animals, serum glucose levels were significantly higher in pre-DM and DM NHPs (Figure 6B, NCD). HCD caused a significant increase in serum glucose in normal animals $(p<0.05)$, but the increase in pre-DM and DM monkeys did not reach statistical significance (Figure 6, HCD, $p>0.05$ ). However, the serum glucose level was significant higher in pre-DM animals than in normal ones (Figure 6B, HCD). Also, the glucose level in DM group under HCD was still statistically significant higher than those in normal and pre-DM groups (Figure 6B, HCD). Except HbA1c significantly higher in DM group than normal and pre-DM groups at the baseline before HCD, other glycemic parameters were not significantly altered 50 days after $\mathrm{HCD}$ (Figure 6C, 6D, 6E).

Feeding the HCD increased serum TC and LDLc by almost 3 folds and in all three experimental groups, which reached statistical difference (Figure 7A, 7D, $p<0.05$ ). HDLc was not significantly changed in all three groups measured at 50 days after HCD (Figure 7C). The TG levels increased in normal and DM groups 50 days after HCD, but the increases did not reach statistical significance compared with NCD (Figure 7B, $p>0.05$ ). However, not like the normal and DM animals, the serum TG level in pre-DM monkeys significantly increased 50 days after HCD (Figure 7B, HCD, $p<0.05$ ).

\section{Dyslipidemia in normal NHPs fed with HCD for a short term}

To investigate the short-term effects of the $\mathrm{HCD}$ and recovery after termination of $\mathrm{HCD}$, a group of normal cynomolgus monkeys ( $\mathrm{n}=6,5$ male, 1 female) were fed with the HCD for 2 months and then washed out for 3 months. Body weight was increased from $8.38 \pm 1.32 \mathrm{~kg}$ before HCD to $9.01 \pm 1.37 \mathrm{~kg} 50$ days after HCD, which was by $8.9 \%$ increase

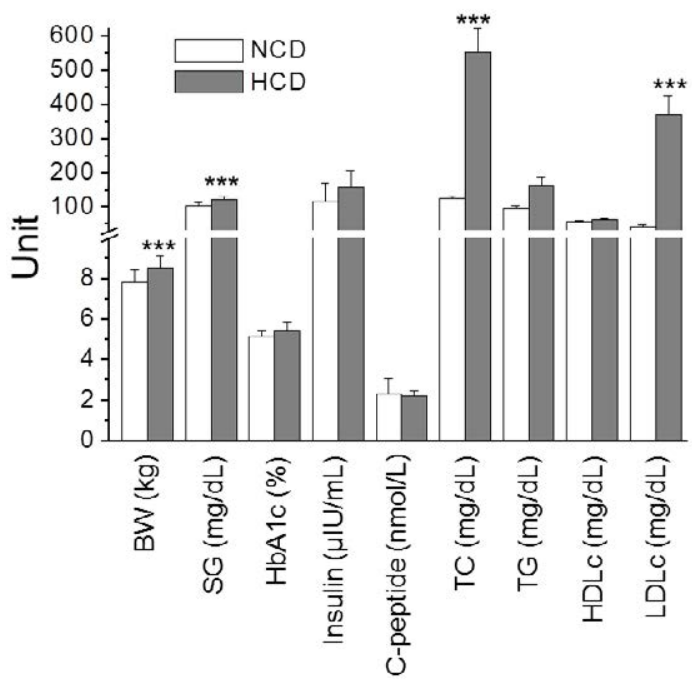

Figure 5: The effects of the HCD on body weight, serum glucose, HbA1c and lipids in a mixed group $(n=30)$ with normal $(n=8)$, pre-DM $(n=11)$ and DM $(n=11)$ NHPs fed with the HCD for 50 days. Compared with the pre-HCD baseline levels (blank bars, NCD), the body weight, serum glucose, HbA1c TC, and LDLC significantly increased 50 days after feeding with the HCD (grey bars, $\mathrm{n}=30$ ). However, HbA1c, insulin, C-peptide, TG and HDL did not significantly change after feeding with the HCD for 50 days $(n=30)$. ${ }^{*}, p<0.05$; ${ }^{* *}, p<0.01 ;{ }^{* *}, p<0.001$; vs. pre-HCD baseline. 
A

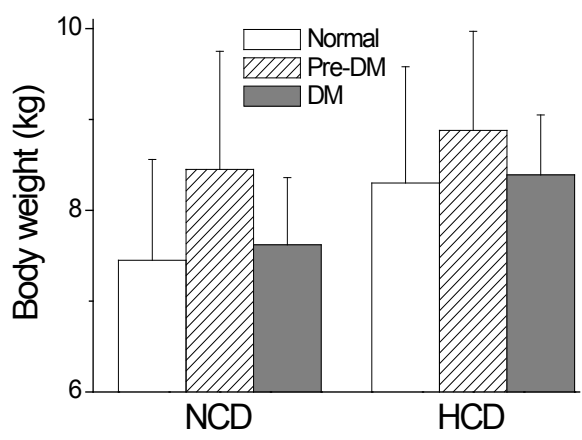

B

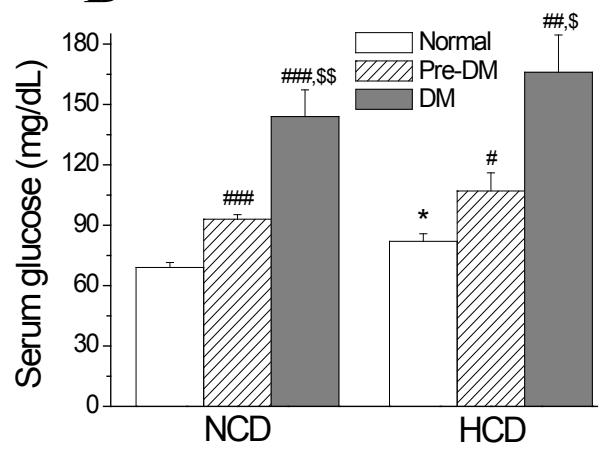

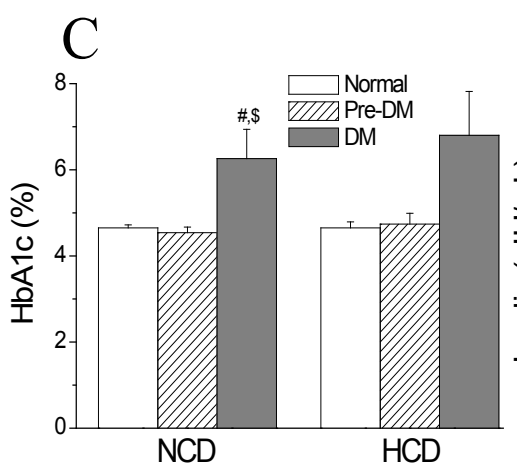

$\mathrm{D}$

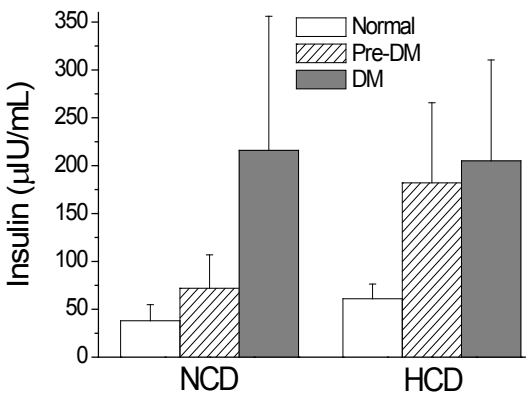

E

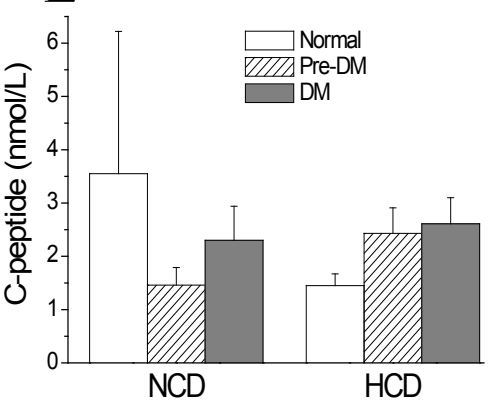

Figure 6: The effects of the HCD on body weight, serum glucose, HbA1c, insulin and C-peptide. $\boldsymbol{A}$, Compared with the NCD, all three groups, normal ( $\mathrm{n}=8$ ), pre-DM $(n=11)$ and DM $(n=11)$, increased their body weights after feeding with the HCD for 50 days, but did not reach statistical significance $(p>0.05)$. However, serum glucose levels significantly increased after feeding with the HCD for 50 days $(\boldsymbol{B})$. HbA1c $(\boldsymbol{C})$, insulin $(\boldsymbol{D})$ and $\mathrm{C}$-peptide $(\boldsymbol{E})$ did not significantly change after feeding with the HCD for 50 days. ${ }^{*}, p<0.05$, vs. NCD baseline; \#, $p<0.05$, \#\#, $p<0.01$, vs. normal; $\$, p<0.05, \$ \$, p<0.01$, vs. pre-DM.
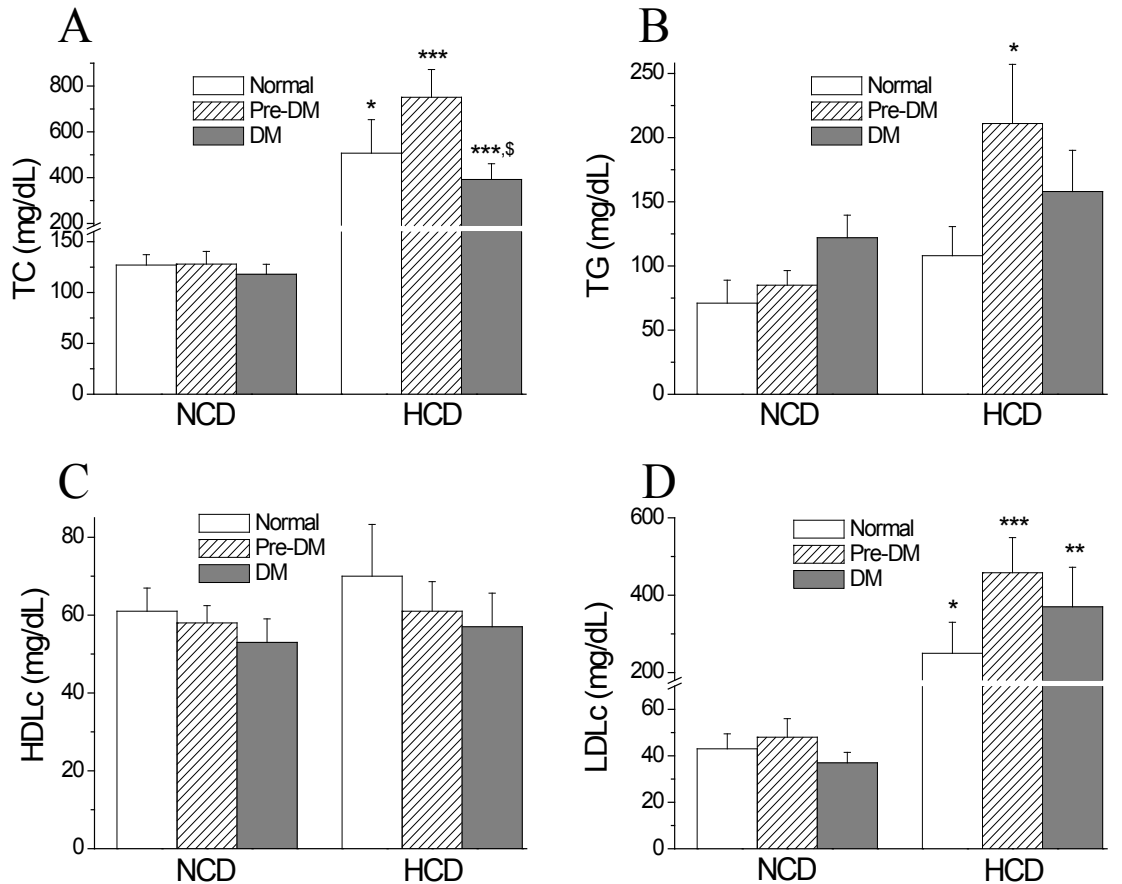

Figure 7: The effects of the HCD on TC, TG, HDLc and LDLc in NHPs. Compared with the NCD, all three groups, normal ( $n=8)$, pre-DM ( $n=11)$ and DM ( $n=11)$, increased their TC $(\boldsymbol{A})$ and LDLc $(\boldsymbol{D})$ levels after feeding with the HCD for 50 days, but did not change TG and HDLc statistically $(p>0.05)$, except TG in pre-DM group (B) after feeding with the HCD for 50 days. ${ }^{*}, p<0.05,{ }^{* *}, p<0.01,{ }^{* * *}, p<0.001$, vs. NCD baseline; $\$, p<0.05$, vs. pre-DM. 
(Figure 8A). After termination of HCD, the body weight was gradually decreased and back to the pre-HCD baseline level in almost a month after switching back to the NCD and then maintained at that level with $\leq 2 \%$ fluctuation (Figure $8 \mathrm{~A}$ ). In the meantime, the serum leptin level was significantly decreased after termination of $\mathrm{HCD}$ (Figure $8 \mathrm{~B}$, $p<0.05)$.

Both serum TC and LDLc levels were significantly increased, from $135 \pm 11 \mathrm{mg} / \mathrm{dL}$ and $41 \pm 6 \mathrm{mg} / \mathrm{dL}$ before HCD to $532 \pm 155 \mathrm{mg} / \mathrm{dL}$ and $442 \pm 83 \mathrm{mg} / \mathrm{dL} 50$ days after HCD (Figure $8 \mathrm{C}, 8 \mathrm{~F}, p<0.05$ ), respectively. However, the changes of serum TG and HDLc were variable and did not reach statistical significance during $\mathrm{HCD}$ and after cessation of the HCD (Figure 8D, 8E, $p>0.05$ ).

\section{Dyslipidemia in obese NHPs with additional HCD}

To investigate the effects of HCD on body weight and other biological effects, a group of animals with total fat above $20 \%$ were selected for the study. The animals were scanned by DXA assay and their averaged total fat $\%$ was $23.6 \pm 1.3 \%(n=4$, male). Then the animals were fed with the HCD for 4 months. Body weight, food consumption, body composition (DXA assay) and blood biochemistry were conducted at various times during the study. Body weight increased slightly after feeding with the HCD for 4 months, from $10.7 \pm 0.1 \mathrm{~kg}$ at day 0 to $11.3 \pm 1.6 \mathrm{~kg}$ at day 120 with the HCD and continuously increased by $0.3 \mathrm{~kg}$ after switching back to the NCD (Figure 9A, $\mathrm{n}=4$ ). Among these animals, \#1 body weight increased so obviously during $\mathrm{HCD}$ and $\# 2$ increased slightly during the late period of HCD.
Animal \#3 body weight decreased initially and then increased slowly during HCD. Obviously \#4 body weight decreased during HCD until switching back to the NCD (Figure 9B). Similar results were observed in another group of pre-HCD obese male monkeys $(n=6)$ and two out of six animals ate very little and lost some of their body weights during 2-month HCD (data not shown due to drug treatment at late stage of HCD). Food consumption showed the changes paralleled to the body weight changes, which increased at the late period of HCD and the time back to NCD (Figure 9C). Several DXA assays (Arrows in Figure 9A) were performed at different time points in the anesthetized animals (10 $\mathrm{mg} / \mathrm{kg}$ ketamine, i.m.). The results showed that the body compositions, including the total fat $\%$ and weights of tissue, fat and lean, changed paralleling to food intake during the study (Figure 9D, $n=4$ ). These data indicate the changes and variabilities of FI, BW and DXA data under NCD or HCD. The changes after HCD were markedly individualized among the animals and responded to the HCD differently.

Blood biochemistry shows that compared with the baseline level before HCD, TC and LDLc increased significantly during HCD and still significantly higher for almost a month after termination of the HCD (Figure 10, $\Delta, \mathbf{\square}, \mathrm{n}=4$ ). Compared with the baseline levels before HCD, blood insulin and TG were higher during the most time of HCD, but none of the parameters at any data points reached statistical significance (Figure $10, \boldsymbol{\bullet}, \boldsymbol{\Delta}, p>0.05, \mathrm{n}=4$ ). However, HDLc levels were lower, but no significance ( $p>0.05$ ) during HCD (Figure 10, $\square, n=4$ ). Blood glucose fluctuation was not so obvious during HCD (Figure 10, $\mathrm{O}, \mathrm{n}=4)$.
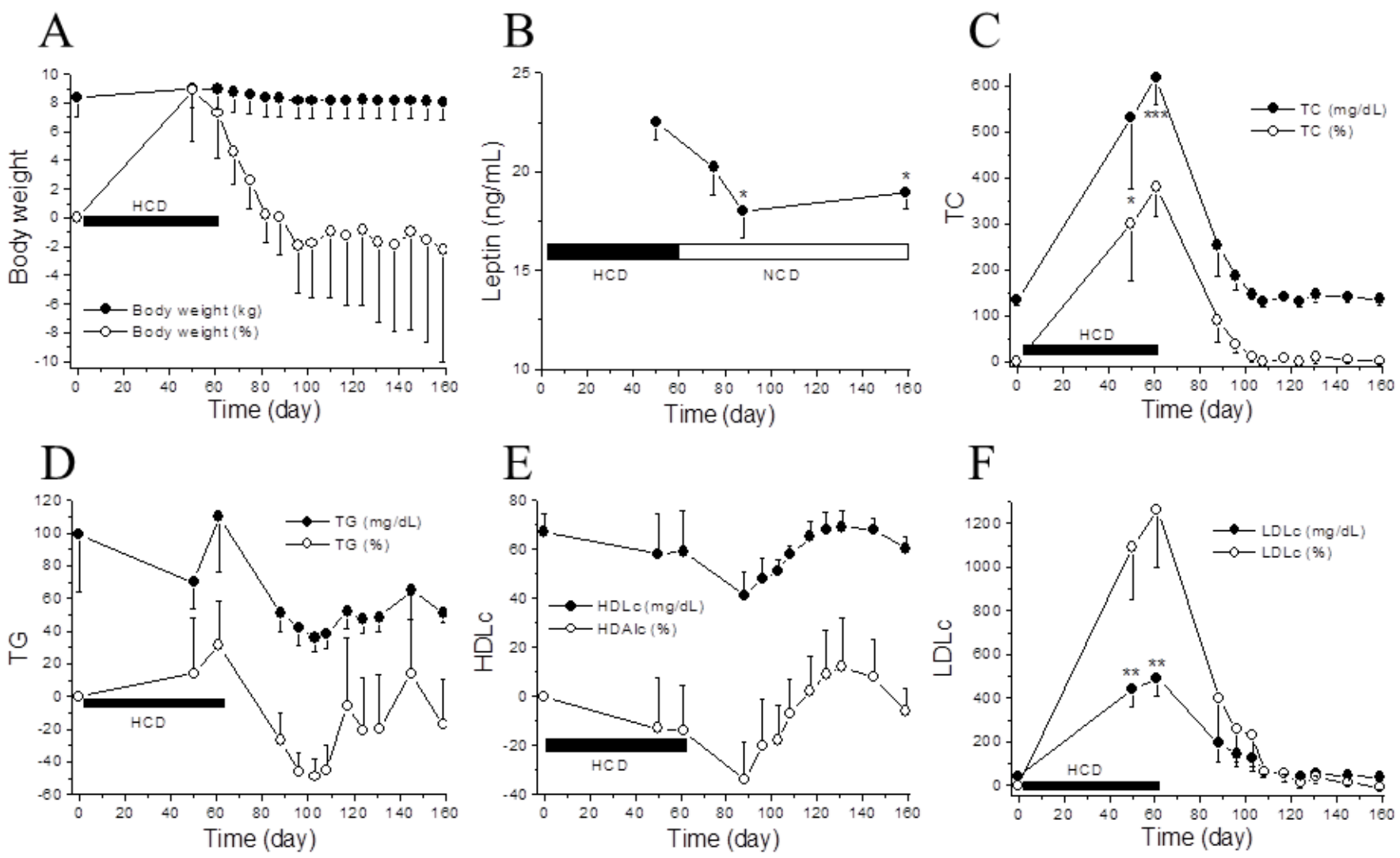

Figure 8: The effects of the HCD on body weight, serum leptin, TC, TG, HDLc and LDLc in normal NHPs $(n=6)$. Compared with the pre-HCD level, body weight increased by $8 \%$ after feeding with the HCD for almost 2-month and returned back to pre-HCD level in a month after switching back to the NCD $(\boldsymbol{A})$. The effects of feeding the HCD for 2 months and washout with the HCD on leptin $(\boldsymbol{B})$, TC $(\boldsymbol{C})$, TG $(\boldsymbol{D}), \mathrm{HDLC}(\boldsymbol{E})$ and LDLc $(\boldsymbol{F})$ are also shown in the corresponding panels. ${ }^{*}, p<0.05$ ${ }^{* *}, p<0.01 ;{ }^{* * *}, p<0.001$; vs. pre-HCD baseline, except leptin in panel $\boldsymbol{B}$, vs. HCD. 
A
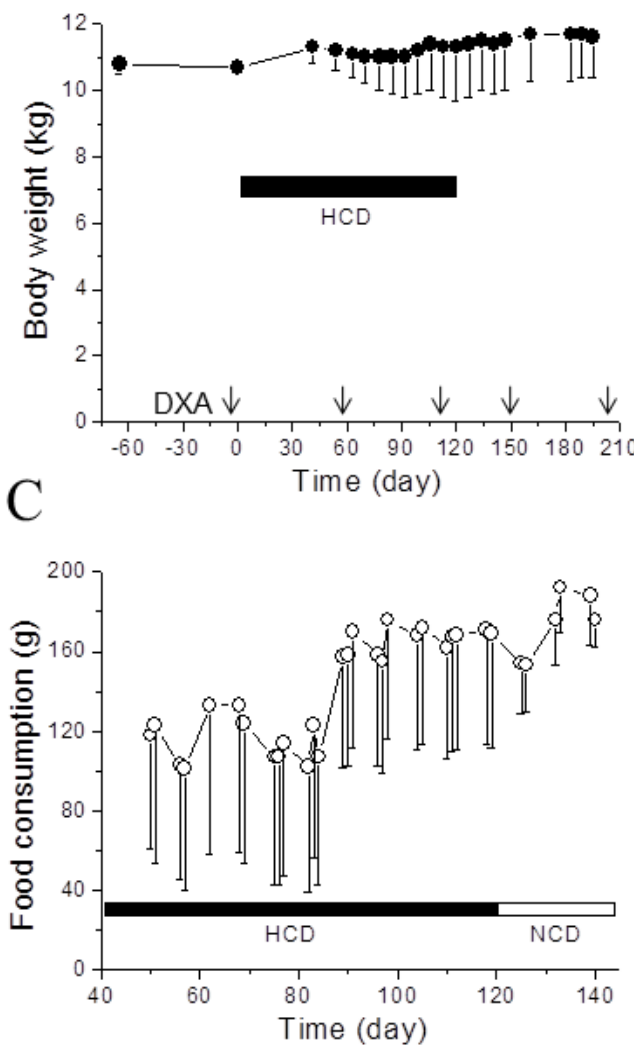

$\mathrm{B}$

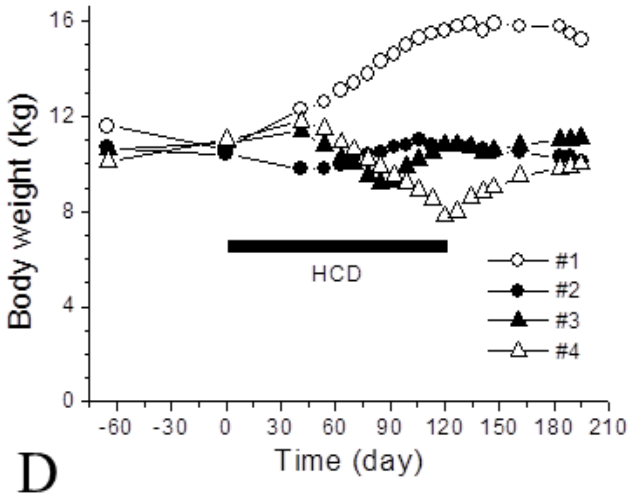

ZIIJ F at \%; $\square$ Tissue (kg); $\square$ Fat (kg);

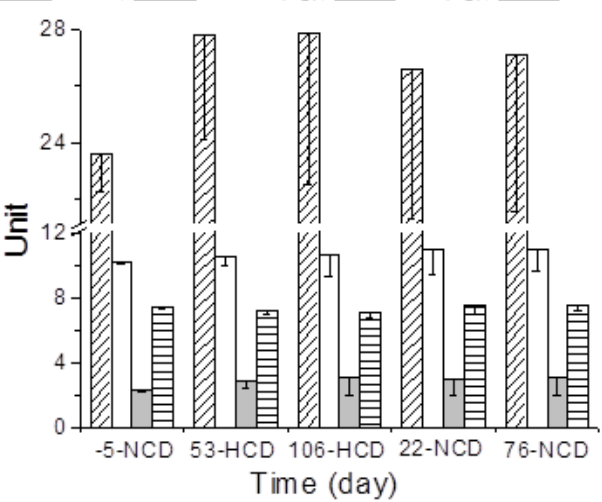

Figure 9: The effects of the HCD on body weight, food intake, and body compositions in the selected pre-HCD obese NHPs ( $n=4)$. Compared with the pre-HCD level, the mean body weight slightly increased $(p>0.05)$ after feeding with the HCD for 4 months in the selected pre-HCD obese NHPs $(\boldsymbol{A})$. The arrows indicated the dates with DAX analysis. However, the increase in body weight was mainly due to one animal $(\boldsymbol{B})$. The averaged food intake (measured twice per week) for the whole group was also slightly increased and such increase was not statistically significant (C). Panel $\boldsymbol{D}$ shows the changes of total fat \% (Fat \%) and weights of tissue (Tissue $(\mathrm{kg})$ ), fat (Fat $(\mathrm{kg})$ ) and lean (Lean $(\mathrm{kg}))$ measured by DXA on the various dates. \#1 to \#4 in panel $\boldsymbol{B}$ were the IDs of animals enrolled in the study. X-axis in panel $\boldsymbol{D}$ : $-5-\mathrm{NCD}=$ day -5 before the HCD; 53-HCD and 106-HCD=day 53 and day 106 after the HCD; 22-NCD and 76-NCD=day 22 and day 76 after switching back to NCD.

\section{Discussion}

This study characterized and summarized the various dyslipidemia and obesity models in our housed NHP cynomolgus macaques (Macaca fascicularis). Cynomolgus monkeys and other strains have been used in many studies for mechanistic research of various diseases and for drug development/test/toxicology, because they have a relatively small body size compared with rhesus monkeys and show various disease models, such as diabetes, obesity, dyslipidemia, cardiovascular diseases and others $[22,33,36]$. These diseases can occur naturally or be induced in housed cynomolgus monkeys via change of their dietary food and/ or increase in their living risk factors, including stress level $[29,36]$. There is a lack of the information to define the normal and obese level in cynomolgus monkeys or other NHPs. In this study the initial characterization and compartmentalizing the levels of NHP obesity are introduced in our housed cynomolgus monkeys (Tables 1 and 2, Figure $1, \mathrm{n}=103)$.

\section{Naturally occurring obesity}

In our housed cynomolgus monkeys, about one third $(29 \%, 30$ out of 103 animals) were lean and all others were obese at various levels according to their DAX data (Table 2, Figure 1). The total body fat $\%$ levels of the animals measured with DXA were uncorrelated with their ages and heights, but were significantly correlated well with their body weights, BMIs, waist circumferences and WHtRs (Figure 2). These obese and dyslipidemic monkeys occurred in our housing facility fed with normal calorie diet. As dyslipidemia and obesity are the increasingly important health issues and have been highly correlated as a risk factor to hyperglycemia and diabetes in humans, animal obesity and diabetes models, especially naturally occurring ones in NHPs, can provide critical research information for understanding potential mechanisms and therapies of obesity, diabetes and their related adverse health issues in humans. Naturally occurring obesity in NHPs has been extensively studied for various purposes, including to develop biomarkers for characterizing overweight individuals [37] and to track the progression of obesity to other complications [22,37]. In this article the naturally developed obese model is introduced and characterized in our housed monkeys with the proposed criteria to group them into various groups based on their body fat composition level (Table 1). Some information on spontaneous obesity and obesityrelated health conditions in cynomolgus monkeys is summarized, which can be useful in obesity research. Similar to overweight humans, obese monkeys increase their risk of cardiovascular complications and other adverse metabolic conditions [22,36,38]. Obesity model in NHPs therefore provide important information relevant to human obesity. Rhesus monkeys raised in indoor cages also exhibit increased rates of obesity, with some of them developing obesity-associated diseases $[28,30,31]$. When given food ad libitum captive macaques display 


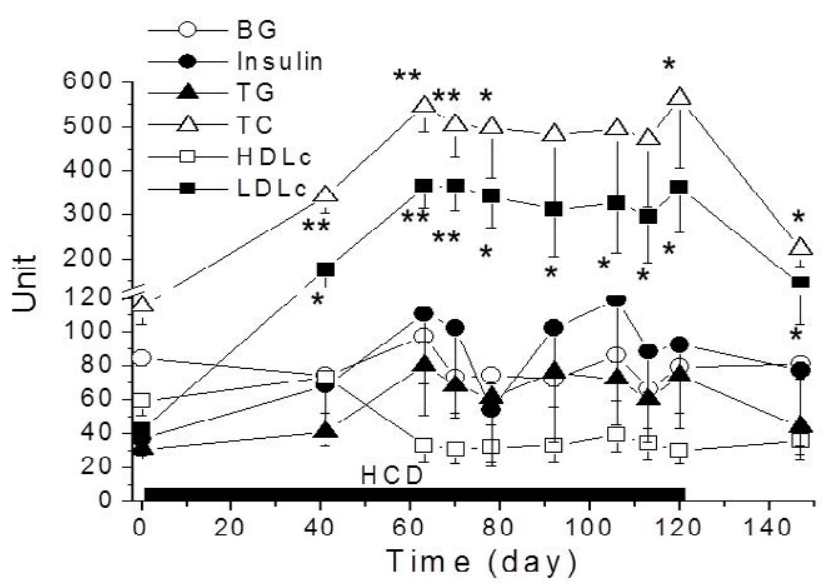

Figure 10: The effects of the HCD on serum glucose, insulin, TC, TG, HDLC and LDLc in selected pre-HCD obese NHPs $(n=4)$. Compared with the preHCD level, TC and LDLc increased significantly during 4-month HCD and even after one month washout with the NCD. However, serum glucose (BG), insulin, TG and HDLc were not significantly changed with more or less variability during HCD. * $p<0.05 ;{ }^{* *}, p<0.01$; vs. pre-HCD baseline.

obesity in an age-dependent manner [33] and develop type 2 diabetes and diabetic complications as humans, likely due to reduced exercise $[32,33,39]$. Spontaneous obesity is also found in wild baboons and in a pedigreed colony $[40,41]$ and occurs in free-ranging rhesus monkeys [42]. Furthermore, a species of Japanese monkey, Macaca fuscata, develops obesity without frank diabetes [43].

\section{Diet-induced obesity}

Clinical studies have shown that increased food intake can result in gaining body weight which can lead to obesity and other related metabolic disorders in humans $[44,45]$. In this study, the HCD (Table 3) was used to induce NHPs' body weight gain, dyslipidemia or obesity as the models for dyslipidemia or obesity research. There was a pattern of the body weight change in the originally normal cynomolgus monkeys $(n=94)$ during over 7 -month HCD feeding. Their body weights were significantly increased within 2 months after HCD and maintained at the increased levels during HCD, even though there was some reduction at the time near the end of HCD and after switching back to the NCD (Figure 3). The body weight changes matched well with daily food intake which gradually increased in the $1^{\text {st }} 2$ months of HCD and then maintained 3 months after HCD feeding (Figure 3). In addition, daily food intake of the NCD markedly decreased at the beginning of HCD termination and then gradually recovered toward the pre-HCD level. That food intake reduction during food switching time could result from the adaption/acclimation to the NCD. The data clearly show that increasing body weight took some time after feeding with the HCD and could be maintained at a high level under HCD. Reduction of the increased body weight also takes time after termination of HCD, even longer than that for HCD-induced gain of the body weight (Figure 3 ). Such phenomenon may involve resetting the food intake and body weight $[46,47]$.

Interestingly, blood glucose levels during HCD were variable, but at a few time points glucose levels increased more obviously in individual animals. However, statistical significance and insignificance were observed compared with the baseline levels during NCD (Figure 3). In contract, compared with control NCD group, the lipid profiles of TC, TG, HDLc and LDLc in HCD animals were significantly increased and even more robustly for TC and LDLc (Figure 3). These results suggest that relatively long-term HCD (7 months) in NHPs could cause significant increase in body weight, TC, TG, HDLc and LDLc, but small change in blood glucose as a whole group, which indicates long-term HCD feeding may not be an effective approach to induce diabetes. However, a relatively short feeding duration (around 4 months) of the HCD in another group of NHPs showed significant increase in body weight, serum glucose, $\mathrm{HbAlc}$, TC and LDLc without obvious changes of TG and HDLc (Figure 4). The data suggest that the outcomes of some biological changes can be varied with the HCD feeding durations.

The HCD effects in NHPs with normoglycemia, pre-DM or DM [35] with 50-day HCD feeding show that as a whole group the body weight, serum glucose, TC and LDLc were significantly increased (Figure 5). However, the effects of HCD on body weight in individual groups, normoglycemia, pre-DM and DM did not reach statistical difference (Figure 6). HCD only caused a significant increase in serum glucose in the normal animals, but not in the pre-DM and DM monkeys (Figure 6). The statistical insignificance might simply be due to the individual variability and/or insufficient animal numbers in each group. HCD increased serum TC and LDLc by almost 3 folds in all three experimental groups (Figure 7). The effects of HCD on metabolism in normoglycemia, pre-DM and DM NHPs were not validated previously. Our data from this study may provide some initial information for those who look at the effects of diet in animals with various metabolism dysfunctions.

Varied biological effects of HCD were also observed in another normal cynomolgus monkey group fed with the HCD for 2 months and then washed out for 3 months (Figure 8). Body weight was increased by $9 \%$ during HCD and gradually decreased back to the pre-HCD level in a month after switching back to NCD with a significant decrease in serum leptin and an increase in TC and LDLc. However, the changes of serum TG and HDLc were variable and no significance.

Variability of biological effects of HCD was also shown in obese monkeys. In selected obese male monkeys (total body fat $=23.6 \pm$ $1.3 \%)$, the effects of HCD on body weight, food consumption and body composition (DXA results) were very individualized with huge differences, even oppositely (some increase, some no change, and some decrease), among the testing animals (Figures 9 and 10). Similar results were observed in another group of six pre-HCD obese male monkeys and two out of six animals ate very little with reduction of their bodywrights during 2-month HCD (data not shown due to drug treatment at late stage of HCD). However, those not eating well with HCD recovered their appetite and body weights after switching back to the NCD, which indicated that those not eating HCD well animals had no health issue, except their poor appetite to the HCD. Such variable responses of FI, BW, DXA and other assay results to the HCD observed in our study are consistent with the data reported previously [48]. Those monkeys responded to high fat diet (HFD) with increasing body weight, plasma lipids, total cholesterol were termed HFD sensitive. The others fed with HFD did not become obese and were defined as HFD resistant. In the previous report plasma FGF21 levels were significantly increased in all HFD fed monkeys compared with the control group. The HFD-sensitive monkeys showed a significant increase in FGF21 mRNA expression in all examined tissues, whereas FGF21 expression in the HFD-resistant group was only increased in the liver, pancreas and the retroperitoneal white adipose tissue (WAT) [48]. In the WAT, the co-receptor $\beta$-klotho was downregulated in the HFD-sensitive monkeys compared with the HFD-resistant group [48].

\section{Limitations of the study}

Our naturally occurring obesity data were collected from over a 
hundred cynomolgus monkeys, but these monkeys were selectively purchased from monkeys farms and raised in cages for a quite long time. Therefore, the distribution and ratio of obesity in these studied animals do not represent any specific epidemic. In addition, the diabetes monkeys were pre-excluded from the monkeys used for naturally obese population in the current study. The occurrence of diabetes did not look through more details according to the obese levels grouped in Table 2. Therefore, the glucose data might not reflect an actual ratio of diabetes monkeys with their corresponding obesity levels. In this study many biochemistry parameters were measured and analyzed (Table 2), but some specific biomarkers, such FGF21 and FFA, were not tested.

In HCD induced-obesity model over one hundred normal monkeys (control, $\mathrm{n}=18$; 7-month HCD, $\mathrm{n}=94$, Figure 3; 4-month HCD, $\mathrm{n}=40$, Figure 4) were enrolled in the study, but for those with pre-DM and $\mathrm{DM}$ the numbers of monkeys were 11, which might not be so powerful to look at the effects of HCD on the diseased models. The same was for those obese monkeys $(n=4)$ treated with the HCD. Therefore, the results from the small groups need to be interpreted with caution. Again, some important biomarkers, such as FGF21, FFA, adiponectin and leptin [49], were unmeasured during the model induction.

\section{Conclusions}

The current data demonstrate that as in humans, natural obesity at various levels occurs in housed cynomolgus monkeys. As their clinical and pathologic characteristics are similar to humans, NHPs have been used for understanding obesity mechanism, pathogenesis, risk factors, comorbidities, and therapeutic interventions. These features are especially critical since many next generation drugs for metabolic diseases also target complications. A naturally occurring NHP obesity model is thus by far the most predictive animal model system for human metabolic syndrome. In addition, this article has summarized HCD-induced obesity model in cynomolgus monkeys housed in our animal facility. In HCD-induced NHP model TC and LDLc significantly increased and stayed at high level stably, but TG increased by less with large variability. Our results also showed that about $30 \%$ obese monkeys were resistant to HCD, which seems higher than normal NHPs. These natural and HCD-induced models in NHPs can be greatly valuable for research and drug therapy of obesity and its associated complications occurred in human diseases.

\section{Author Details}

All the authors, except Keefe Chng, are the employees of Cardiovascular and Metabolic Disease Research, Crown Bioscience Inc., 6 Beijing West Road, Taicang, Jiangsu Province, China. Keefe Chng is the employee of Crown Bioscience North Carolina (CBNC), 150 N Research Campus Drive, Kannapolis, NC, USA.

\section{References}

1. Pollack A (2013) Recognizes Obesity as a Disease. New York Times.

2. (2011) Combating the global obesity epidemic. J Diabetes 3: 7-8, 13-4.

3. Organization. WH: (2011) Obesity and overweight: factsheet No311.

4. Lau DC, Douketis JD, Morrison KM, Hramiak IM, Sharma AM, et al. (2007) 2006 Canadian clinical practice guidelines on the management and prevention of obesity in adults and children [summary]. CMAJ 176: S1-13.

5. Bleich S, Cutler D, Murray C, Adams A (2008) Why is the developed world obese? Annu Rev Public Health 29: 273-295.

6. Haslam DW, James WP (2005) Obesity. Lancet 366: 1197-1209.

7. Guh DP, Zhang W, Bansback N, Amarsi Z, Birmingham CL, et al. (2009) The incidence of co-morbidities related to obesity and overweight: a systematic review and meta-analysis. BMC Public Health 9: 88.
8. Brown WV, Fujioka K, Wilson PW, Woodworth KA (2009) Obesity: why be concerned? Am J Med 122: S4-11.

9. Muoio DM, Newgard CB (2008) Mechanisms of disease:Molecular and metabolic mechanisms of insulin resistance and beta-cell failure in type 2 diabetes. Nat Rev Mol Cell Biol 9: 193-205.

10. Forbes JM, Cooper ME (2013) Mechanisms of diabetic complications. Physiol Rev 93: 137-188.

11. Scicchitano P, Cameli M, Maiello M (2014) Nutraceuticals and dyslipidaemia: Beyond the common therapeutics. Journal of Functional Foods 6: 11-32.

12. King AJ (2012) The use of animal models in diabetes research. Br J Pharmacol 166: 877-894.

13. Sasase T, Pezzolesi MG, Yokoi N, Yamada T, Matsumoto K (2013) Animal models of diabetes and metabolic disease. J Diabetes Res 2013: 281928.

14. Chatzigeorgiou A, Halapas A, Kalafatakis K, Kamper E (2009) The use of animal models in the study of diabetes mellitus. In Vivo 23: 245-258.

15. Islam MS (2013) Animal models of diabetic neuropathy: progress since 1960s. J Diabetes Res 2013: 149452.

16. Rees DA, Alcolado JC (2005) Animal models of diabetes mellitus. Diabet Med 22: $359-370$

17. Hsueh W, Abel ED, Breslow JL, Maeda N, Davis RC, et al. (2007) Recipes for creating animal models of diabetic cardiovascular disease. Circ Res 100: 1415-1427.

18. Wagner JD, Cline JM, Shadoan MK, Bullock BC, Rankin SE, et al. (2001) Naturally occurring and experimental diabetes in cynomolgus monkeys: a comparison of carbohydrate and lipid metabolism and islet pathology. Toxicol Pathol 29: 142-148.

19. Harwood HJ Jr, Listrani P, Wagner JD (2012) Nonhuman primates and other animal models in diabetes research. J Diabetes Sci Technol 6: 503-514.

20. Wagner JE, Kavanagh K, Ward GM, Auerbach BJ, Harwood HJ Jr, et al. (2006) Old world nonhuman primate models of type 2 diabetes mellitus. ILAR J 47: 259-271.

21. Zhu H, Yu L, He Y, Wang B (2014) Nonhuman primate models of type 1 diabetes mellitus for islet transplantation. J Diabetes Res 2014: 785948.

22. Wang X, Wang B, Sun G, Wu J, Liu Y, et al. (2015) Dysglycemia and Dyslipidemia Models in Nonhuman Primates: Part I. Model of Naturally Occurring Diabetes. J Diabetes Metab S13: 010

23. Liu Y, Wang B, Sun G, Ding X, Wang X, et al. (2015) Dynamic Changes of Blood Betatrophin, Glucose, and Insulin during STZ Induction of Diabetes in Rhesus Monkeys. American Diabetes Association 75th Scientific Sessions Abstract 2355-P

24. Xiao YF, Wang B, Wang X, Du F, Benzinou M, et al. (2013) Xylazine-induced reduction of tissue sensitivity to insulin leads to acute hyperglycemia in diabetic and normoglycemic monkeys. BMC Anesthesiol 13: 33 .

25. Guo S, Qian W, Du F, Wang B, Wang X, et al. (2014) Proteinuria in Cynomolgus macaques (Macaca fascicularis) with Spontaneously Developed Metabolic Disorder and Diabetes: Transcriptome Analysis of Biopsy Kidney. Journal of Diabetes \& Metabolism 5: 334.

26. Wang X, Hansen BC, Shi D, Fang Y, Du F, et al. (2013) Quantification of betacell insulin secretory function using a graded glucose infusion with $\mathrm{C}$-peptide deconvolution in dysmetabolic, and diabetic cynomolgus monkeys. Diabetol Metab Syndr 5: 40.

27. Page SL, Goodman M (2001) Catarrhine phylogeny: noncoding DNA evidence for a diphyletic origin of the mangabeys and for a human-chimpanzee clade. Mol Phylogenet Evol 18: 14-25.

28. Hansen BC, Bodkin NL (1986) Heterogeneity of insulin responses: phases leading to type 2 (non-insulin-dependent) diabetes mellitus in the rhesus monkey. Diabetologia 29: 713-719.

29. Kanasaki K, Koya D (2011) Biology of obesity: lessons from animal models of obesity. J Biomed Biotechnol 2011: 197636.

30. Bodkin NL, Ortmeyer HK, Hansen BC (1993) Diversity of insulin resistance in monkeys with normal glucose tolerance. Obes Res 1: 364-370.

31. Bodkin NL, Hannah JS, Ortmeyer HK, Hansen BC (1993) Central obesity in 
Citation: Wang B, Sun G, Liu Y, Qiao J, Ye W, et al. (2016) Dysglycemia and Dyslipidemia Models in Nonhuman Primates: Part II. Model of Naturally Occurring or Experimental Obesity. J Diabetes Metab 7: 641. doi:10.4172/2155-6156.1000641

Page 11 of 11

rhesus monkeys: association with hyperinsulinemia, insulin resistance and hypertriglyceridemia? Int J Obes Relat Metab Disord 17: 53-61.

32. Hansen BC, Bodkin NL (1993) Primary prevention of diabetes mellitus by prevention of obesity in monkeys. Diabetes 42: 1809-1814.

33. Kemnitz JW (1984) Obesity in macaques: spontaneous and induced. Adv Vet Sci Comp Med 28: 81-114.

34. St-Onge MP, Wang J, Shen W, Wang Z, Allison DB, et al. (2004) Dual-energy $x$-ray absorptiometry-measured lean soft tissue mass: differing relation to body cell mass across the adult life span. J Gerontol A Biol Sci Med Sci 59: 796-800.

35. Wang X, Wang B, Sun G, Wu J, Liu Y, et al. (2015) Dysglycemia and Dyslipidemia Models in Nonhuman Primates: Part I. Model of Naturally Occurring Diabetes. J Diabetes Metab S13: 010.

36. Gu H, Liu Y, Mei S, Wang B, Sun G, et al. (2015) Left ventricular diastolic dysfunction in nonhuman primate model of dysmetabolism and diabetes. BMC Cardiovasc Disord 15: 141

37. Bauer SA, Arndt TP, Leslie KE, Pearl DL, Turner PV (2011) Obesity in rhesus and cynomolgus macaques: a comparative review of the condition and its implications for research. Comp Med 61: 514-526.

38. Standaert ML, Ortmeyer HK, Sajan MP, Kanoh Y, Bandyopadhyay G, et al. (2002) Skeletal muscle insulin resistance in obesity-associated type 2 diabetes in monkeys is linked to a defect in insulin activation of protein kinase C-zeta/ lambda/iota. Diabetes 51: 2936-2943.

39. Hansen BC, Ortmeyer HK, Bodkin NL (1995) Prevention of obesity in middleaged monkeys: food intake during body weight clamp. Obes Res 3 Suppl 2: 199s-204s.

40. Banks WA, Phillips-Conroy JE, Jolly CJ, Morley JE (2001) Serum leptin levels in wild and captive populations of baboons (papio): implications for the ancestral role of leptin. J Clin Endocrinol Metab 86: 4315-4320.

41. Banks WA, Altmann J, Sapolsky RM, Phillips-Conroy JE, Morley JE (2003) Serum leptin levels as a marker for a syndrome X-like condition in wild baboons. J Clin Endocrinol Metab 88: 1234-1240.

42. Schwartz SM, Kemnitz JW, Howard CF Jr (1993) Obesity in free-ranging rhesus macaques. Int J Obes Relat Metab Disord 17: 1-9.

43. Takahashi T, Higashino A, Takagi K, Kamanaka Y, Abe M, et al. (2006) Characterization of obesity in Japanese monkeys (Macaca fuscata) in a pedigreed colony. J Med Primatol 35: 30-37.

44. Drewnowski A, Kurth C, Holden-Wiltse J, Saari J (1992) Food preferences in human obesity: carbohydrates versus fats. Appetite 18: 207-221.

45. Krahn D, Kurth C, Demitrack M, Drewnowski A (1992) The relationship of dieting severity and bulimic behaviors to alcohol and other drug use in young women. J Subst Abuse 4: 341-353.

46. Ortega-Molina A, Lopez-Guadamillas E, Mattison JA, Mitchell SJ, MunozMartin M, et al. (2015) Pharmacological inhibition of PI3K reduces adiposity and metabolic syndrome in obese mice and rhesus monkeys. Cell Metab 21: 558-570.

47. Shin AC, Berthoud HR (2013) Obesity surgery: happy with less or eternally hungry? Trends Endocrinol Metab 24: 101-108.

48. Nygaard EB, Møller CL, Kievit P, Grove KL, Andersen B (2014) Increased fibroblast growth factor 21 expression in high-fat diet-sensitive non-human primates (Macaca mulatta). Int J Obes (Lond) 38: 183-191.

49. Chen Y, Ogawa H, Narita H, Ohtoh K, Yoshida T, et al. (2003) Ratio of leptin to adiponectin as an obesity index of cynomolgus monkeys (Macaca fascicularis). Exp Anim 52: 137-143. 